and sex, and diagnose gout with a sensitivity of $95.1 \%$, summarized in Figure 1 . Linear regressions demonstrated an elevated CRP response in people suffering from type II diabetes, higher cumulative joint points score, number of affected joints, as well as elevated uric acid, ESR, and leucocyte count.

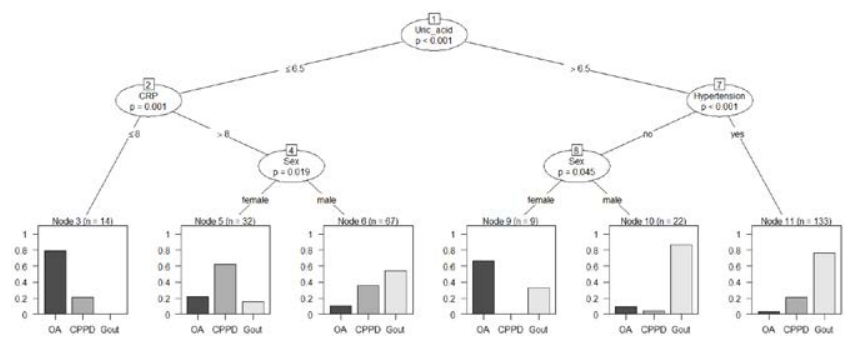

Figure 1. Conditional inference tree using unbiased recursive partitioning to reliably differentiate between gout, osteoarthritis, and calcium pyrophosphate deposition disease.

Conclusion: Gout can be reliably diagnosed, simultaneously excluding $\mathrm{OA}$ and CPPD as differential diagnoses by conditional inference trees using just four biomarkers. A correlation between inflammatory reaction severity based on CRP levels was found in patients suffering from type II diabetes, more or larger joint involvement and elevated uric acid levels. The double contour sign remains a questionable differentiator between gout and CPPD with a sensitivity/specificity of $71 \% / 55 \%$ for gout and $59 \% / 39 \%$ for CPPD, similar to findings reported by Löffler et al (1) with a sensitivity/specificity of only $64 \% / 52 \%$ for gout.

REFERENCES:

[1] Löfler C, Sattler H, Peters L, Löffler U, Uppenkamp M, Bergner R. Distinguishing gouty arthritis from calcium pyrophosphate disease and other arthritides. J Rheumatol 2015; 42(3):513-20.

Disclosure of Interests: Dmitrij Kravchenko Shareholder of: Pfizer, Raoul Bergner: None declared, Charlotte Behning: None declared, Valentin Schäfer Speakers bureau: AbbVie, Novartis, BMS, Chugai, Celgene, Medac, Sanofi, Lilly, Hexal, Pfizer, Janssen, Roche, Schire, Onkowissen, Royal College London, Consultant of: Novartis, Chugai, AbbVie, Celgene, Sanofi, Lilly, Hexal, Pfizer, Amgen, BMS, Roche, Gilead, Medac, Grant/research support from: Novartis, Hexal, Lilly, Roche, Celgene, Universität Bonn.

DOI: 10.1136/annrheumdis-2021-eular.1855

\section{POS1135 MONOSODIUM URATE CRYSTALS REDUCE SCHWANN CELLS VIABILITY}

Y. Liu ${ }^{1,2}$, Y. Huang ${ }^{1}$, S. Sun ${ }^{1}$, W. Deng ${ }^{1}$, T. W. LI ${ }^{1} .{ }^{1}$ Guangdong Second Provincial General Hospital, Department of Rheumatology and Immunology, Guangzhou, China; ${ }^{2}$ University of South China, Guangdong Second Provincial General Hospital, Guangzhou, China

Background: The prevalence of peripheral neuropathy in patients with gout almostly reaches $25 \%$ [1]. Demyelination caused by Schwann cell (SCs) injury and apoptosis is the major pathological feature of peripheral[2]. None of study has focused on the effects of monosodium urate (MSU) crystals on SCs.

Objectives: To assess the effect of MSU crystals on SCs.

Methods: Mouse-derived Schwann cells (RSC96) are stimulated with different concentrations of MSU crystals $(0 \mathrm{mg} / \mathrm{ml}, 0.25 \mathrm{mg} / \mathrm{ml}, 0.5 \mathrm{mg} / \mathrm{ml})$ and time $(24 \mathrm{~h}, 48 \mathrm{~h}, 72 \mathrm{~h})$. The migration ability of Schwann cells is evaluated by acratch assay, the proliferation level is assessed by the cell counting kit-8 (CCK-8) assay, and the apoptosis rate is detected by flow cytometry.

Results: The acratch assay showed that the migration ability of SCs was worsened, CCK-8 assay suggested that proliferation of SCs was reduced in a dose-dependent manner $(P<0.05)$. According to the result of flow cytometry, the survival rate of SCs at $0.5 \mathrm{mg} / \mathrm{ml}(78.60 \% \pm 2.26 \%)$ was lower than that $0.25 \mathrm{mg} /$ $\mathrm{ml}(87.50 \% \pm 0.95 \%)$ and $0 \mathrm{mg} / \mathrm{ml}(98.80 \% \pm 0.26 \%)(\mathrm{p}<0.05)$ at $24 \mathrm{~h}$. When the stimulation time increased to $72 \mathrm{~h}$, the survival rate at $0.5 \mathrm{mg} / \mathrm{ml}(47.90 \% \pm 11.70 \%)$ dropped significantly, which was significantly different from the other two groups $(\mathrm{p}<0.05)$.

Conclusion: MSU crystals can cause damage to Schwann cells. It may help to explain the reason of peripheral neuropathy in gout patients.

REFERENCES:

[1] López-López, C.O., et al., Peripheral neuropathies in rheumatic diseases: More diverse and frequent than expected. A cross-sectional study. International journal of rheumatic diseases, 2020. 23(2): p. 226-232.

[2] Liu, Y., S. Shao and H. Guo, Schwann cells apoptosis is induced by high glucose in diabetic peripheral neuropathy. Life sciences, 2020. 248: p. 117459.

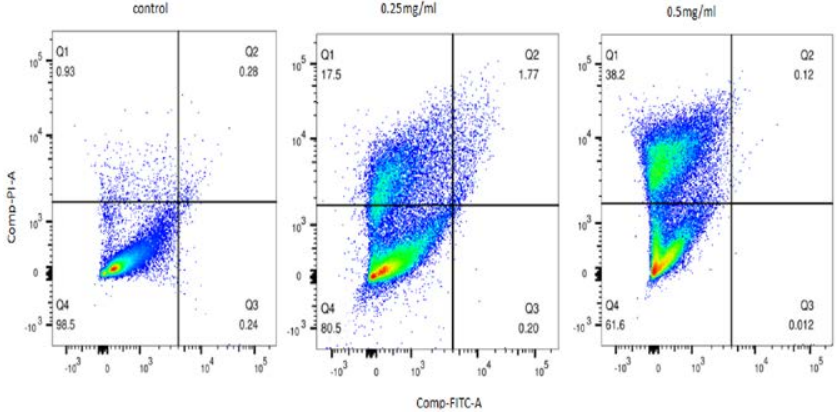

Figure 1. Flow cytometry assays of RSC96 on MSU crystals at 72h

Disclosure of Interests: None declared.

DOI: 10.1136/annrheumdis-2021-eular.1989

\section{POS1136 PHARMACOKINETICS OF PEGLOTICASE AND METHOTREXATE POLYGLUTAMATE(S) IN PATIENTS WITH UNCONTROLLED GOUT RECEIVING PEGLOTICASE AND CO-TREATMENT OF METHOTREXATE}

Y. Song ${ }^{1}$, Y. Xin ${ }^{1}$, M. E. Weinblatt ${ }^{2}$, J. Chamberlain ${ }^{1}$, K. Obermeyer ${ }^{3}$, L. Zhao ${ }^{3}$, C. Canavan ${ }^{3}$, P. M. Peloso ${ }^{3}$, S. Ramanathan ${ }^{1} .{ }^{1}$ Horizon Therapeutics plc, Research and Development, South San Francisco, United States of America; ${ }^{2}$ Brigham and Women's Hospital, Division of Rheumatology, Immunology and Allergy, Boston, United States of America; ${ }^{3}$ Horizon Therapeutics plc, Research and Development, Deerfield, United States of America

Background: In an open-label, single-arm trial in adult patients with uncontrolled gout (MIRROR open-label [OL] trial) evaluating pegloticase co-treatment with methotrexate (MTX); $78.6 \%$ patients were responders, defined as maintenance of serum uric acid $<6 \mathrm{mg} / \mathrm{dL}$ for at least $80 \%$ of the time during month 6 [weeks 20, 22, and 24]. In comparison, $42 \%$ patients achieved a response during month 3 and 6 in historical Phase 3 monotherapy trials of pegloticase (C0405 and C0406) ${ }^{1}$. MTX co-treatment is shown to improve the pharmacokinetics (PK) of biologics by attenuating the formation of anti-drug antibodies ${ }^{2}$.

Objectives: To determine the systemic exposures of pegloticase and methotrexate polyglutamate(s) (MTX-PGs) in uncontrolled gout patients receiving pegloticase and MTX; to evaluate the effect of MTX on the PK of pegloticase in comparison to historical pegloticase monotherapy trials (C0405 and C0406) ${ }^{4}$; and to evaluate the immunogenicity of pegloticase in co-treatment with MTX. Methods: In the MIRROR OL trial, MTX (15 mg/week) was given orally 4 weeks prior to the first pegloticase dose and continued weekly, in combination with pegloticase $8 \mathrm{mg}$ given intravenously every 2 weeks, for a treatment duration of up to 52 weeks. Pre-infusion samples were collected to measure MTXPGs in red blood cells. Pre- and post-infusion blood samples were obtained to measure the peak $\left(\mathrm{C}_{\mathrm{mx}}\right)$ and trough $\left(\mathrm{C}_{\text {min }}\right)$ concentrations of pegloticase at multiple visits. Anti-drug antibody blood samples were collected at multiple visits. The impact of MTX on pegloticase PK was evaluated by comparing pegloticase exposures with MTX from this trial to historical monotherapy data $(\mathrm{C} 0405 \text { and } \mathrm{C} 0406)^{3,4}$. The observed pegloticase concentrations with MTX were also overlaid with the $90 \%$ prediction interval based on the population PK mode $\left.\right|^{5}$ from $\mathrm{C} 0405$ and C0406.

Results: Pegloticase and MTX-PG levels were determined in 14 patients. The 11 responders were generally associated with higher pegloticase exposures than the non-responders, especially $C_{\text {nig }}$ (Figure 1). Concomitant treatment of MTX resulted in fewer patients with $C_{\text {min }}$ below quantitation limit (BQL) (5/14 [36\%] with MTX vs $63 / 82[77 \%]$ without MTX), and higher overall $C_{\min }$ (median: $1.03 \mu \mathrm{g} / \mathrm{ml}$ with MTX vs BQL without MTX); C $_{\text {max }}$ was slightly higher (median [Q1, Q3]: 2.11 [1.65, 2.59] $\mu \mathrm{g} / \mathrm{mL}$ with MTX vs 1.51 [BQL, 2.48] $\mu \mathrm{g} / \mathrm{mL}$ without MTX). Pegloticase co-treatment with MTX resulted in more concentrations above the predicted median value of pegloticase, compared to monotherapy. ADA data is consistent with pegloticase PK and efficacy. Significant increase in ADA titers were only observed in 2 subjects (both were non-responders) at time corresponding to the loss of pegloticase exposure and increases in sUA levels. Concentrations of MTX-PGs were maintained during the treatment course, suggesting compliance of MTX administration. There was no apparent difference in concentrations of MTX-PGs between responders and non-responders.

Conclusion: Pegloticase $8 \mathrm{mg}$ IV every 2 weeks co-treatment with MTX $15 \mathrm{mg}$ weekly resulted in fewer patients with pegloticase $C_{\min }$ below the quantification 